\title{
Antioxidants: Potential Inducers of Gene Expression through CAMP Dependent Pathway
}

Samarth Tandon*, Aayush Kukreja, Amit Mishra and Archana Tiwari

School of Biotechnology, Rajiv Gandhi Proudyogiki Vishwavidyalaya-Bhopal-462033-Madhya Pradesh, India

\begin{abstract}
Antioxidants have played a pivotal role in the treatment of various diseases like cancer, diabetes, cardiovascular diseases. The major goal of this review is to hypothesize the treatment strategy by the use of antioxidants for correcting the molecular defects found in $\beta$-thalassemia major. In that vein, we are trying to determine the mechanism of action and the role of two most potent antioxidants viz. Lycopene and Betalain in correcting the molecular defects of $\beta$-thalassemia major. The strategy discussed here can be utilized for the purpose of treatment of molecular anomalies in $\beta$-thalassemia major, which is on the line of enhancing the $Y$-globin gene expression by augmenting the cAMP level in the erythroid cells.
\end{abstract}

Keywords: Antioxidant; cAMP; Lycopene; Betalain; $\beta$-thalassemia

\section{Introduction}

Thalassemia is an inbred hemolytic disorder caused by a partial or complete deficiency of $\alpha$ or $\beta$ chain synthesis. The protein hemoglobin is mainly composed of Heme and Globin. Among them heme is a prosthetic group which functions in promoting the reversible binding of oxygen with hemoglobin and globin is the protein which functions in surrounding and protecting the heme part of hemoglobin. Globin the protein part is mainly comprised of two chains viz. $\alpha$-chain and $\beta$-chain, upon the exception of the very first week of embryogenesis, one of the globin chains is always $\alpha$ and the other non- $\alpha$ chain is designated as $\gamma$-chain [1].

The genetic control of globin chain synthesis is primarily accomplished by two developmentally synchronized gene clusters; these are located on the chromosome band 16p13.3 and 11p15.5 (for alpha and non-alpha gene cluster, respectively) [2]. In humans there are eight distinct genetic loci that code for the six types of globin chains. In addition there are minimum of four pseudo genes that have sequences similar to other globin genes but are not expressed into globin proteins. In the $\beta$-globin gene cluster, the sequences around the gene appear to carry some developmental specificity and the genes on the human $\beta$-globin cluster are arranged in their temporal expression during development [3].

The genes in each globin cluster are aligned in 5' 3' direction on the coding strands in the order of their temporal developmental expression during embryonic, fetal and postnatal life [4]. There are five functional genes: $5^{\prime}-\varepsilon^{-}{ }^{\mathrm{G}} \gamma-{ }^{\mathrm{A}} \gamma-\Psi \beta-\delta-\beta-3$ '. Balanced gene expression is obligatory for normal red cell function. Interference of the equilibrium produces a disorder called thalassemia [5].

The gene imbalance can up to some extent be regulated by various secondary messengers based pathways like cAMP dependent pathway, MAPK dependent pathway, p38 kinase dependent pathway, these pathways are regulated by the level of various cytokines and antioxidants like Epo., SCF, TGF- $\beta$ and Forskolin, Lycopene, Betalain etc [6].

Here in this article our prime focus will be on the cAMP dependent pathway and the role of antioxidants in its augmentation which further leads to the increased expression of $\gamma$-globin gene.

\section{cAMP dependent pathway}

Cyclic adenosine monophosphate (cAMP or 3'-5'-cyclic adenosine monophosphate) is a secondary messenger important in many biological processes for example, cAMP is implicated in memory consolidation, immune function, regulation of insulin secretion, and cardiac frequency [7]. Future research is nowadays concentrated on the role of cAMP in $\beta$-thalassemia patients (Figure 1).

cAMP is derived from adenosine triphosphate (ATP) and used for intracellular signal transduction in many different organisms, conveying the cAMP-dependent pathway. cAMP is synthesized from ATP by the action of the key enzyme adenylyl cyclase located on the inner side of the plasma membrane. Activation of adenylyl cyclase take place by a range of signaling molecules through the activation of adenylyl cyclase stimulatory $\mathrm{G}\left(\mathrm{G}_{\mathrm{s}}\right)$-protein-coupled receptors and inhibited by agonists of adenylyl cyclase inhibitory $G\left(G_{i}\right)$-proteincoupled receptors. cAMP decomposition into AMP is catalyzed by the enzyme phosphodiesterase [8]. cAMP, either via a PKA(Protein Kinase A) dependent or PKA independent manner, affects numerous cellular functions and can exert different biological effects such as cell differentiation, proliferation and apoptosis.

Therefore, a major question for scientists working in the field of the cAMP signaling has been to understand how specificity is maintained in this second messenger system. As for example elevation of cAMP inhibits the proliferation and expression of transformed phenotype in a wide variety of cancer models, including breast cancer, through the induction of apoptosis and/or cell cycle arrest [9]. Moreover cAMP as a secondary messenger also plays a key role in regulating the blood glucose level through glycogen metabolism which has positive effects in diabetes [8].

In patients with $\beta$-thalassemia intermedia, cAMP levels were found to be elevated in both red blood cells and nucleated erythroblasts,

*Corresponding author: Samarth Tandon, School of Biotechnology, Rajiv Gandhi Proudyogiki Vishwavidyalaya-Bhopal-462033-Madhya Pradesh, India, E-mail id: tandon_samarth@hotmail.com

Received April 16, 2013; Accepted August 07, 2013; Published August 09, 2013

Citation: Tandon S, Kukreja A, Mishra A, Tiwari A (2013) Antioxidants: Potential Inducers of Gene Expression through CAMP Dependent Pathway. J Blood Disorders Transf S3: 006. doi:10.4172/2155-9864.S3-006

Copyright: $\odot 2013$ Tandon S, et al. This is an open-access article distributed under the terms of the Creative Commons Attribution License, which permits unrestricted use, distribution, and reproduction in any medium, provided the original author and source are credited. 
cAMP-dependent pathway efficiently induced $\gamma$-globin expression in adult erythroblasts, and this pathway plays a role in $\gamma$-globin gene expression in $\beta$-thalassemia. Many antioxidants like Forskolin, Lycopene, betalain etc. may play a key role in inducing the $\gamma$-globin gene expression through cAMP dependent pathway [6].

\section{Antioxidants}

Transfer of electron from electron rich to electron scarce entity is termed as oxidation reaction [10]. Antioxidant is a compound capable of preventing or slowing the oxidation of other molecules. Usually, an antioxidant protects against metal toxicity by trapping free radicals thus terminating the chain reaction, by chelating metal ion and preventing the reaction with reactive oxygen species(ROS) or by chelating metal and maintaining it in a redox state leading to its incompetency to reduce molecular oxygen [11] (Figure 2).

The molecules with unpaired electrons in their outer shell are termed as free radicals; they participate in origin of life and biological evolution, leaving beneficial effects on the organisms [12]. Reactive oxygen species (ROS) are chemically reactive molecules containing oxygen for example oxygen ions and peroxides. ROS form as a natural byproduct of the normal metabolism of oxygen and have important roles in cell signaling and homeostasis [13].

Reactive oxygen species (ROS) and Reactive Nitrogen Species (RNS) are also the by-products of mitochondria-catalyzed electron transport reactions and other mechanisms [11].

ROS at higher concentration are important mediators of damage to cell structures, including lipids and membranes, proteins and nucleic acids (termed oxidative stress). The harmful effects of ROS are balanced by the antioxidant action of non-enzymatic antioxidants in addition to antioxidant enzymes [14].

\section{Nomenclature and classification of antioxidants}

Antioxidants are broadly classified in two categories viz. antioxidant enzymes and non enzymatic antioxidants. Non-enzymatic antioxidants as well as antioxidant enzymes are known to neutralize the effect of ROS and RNS. These antioxidants are known to disseminate free radicals leading to limited risk of oxidative stress. At cellular and molecular level they inactivate ROS and under specific low concentration inhibit or delay oxidative processes by interrupting the radical chain reaction. Antioxidants also chelate the metal ions responsible for the generation of ROS as they have the potentials to work in both aqueous and/or membrane domains [15].

Overproduction of free radicals and reactive oxygen species can cause oxidative damage to biomolecules, (lipids, proteins, DNA), eventually leading to many chronic diseases such as atherosclerosis, cancer, diabetics, rheumatoid arthritis, post-ischemic perfusion injury, myocardial infarction, cardiovascular diseases, chronic inflammation, stroke and septic shock, aging and other degenerative diseases in humans [16].

\section{Treatment with antioxidants}

This study is mainly focused on the two non enzymatic antioxidants viz. Lycopene and Betalain (Figure 3 and 4). Among them lycopene is a red pigment that occurs naturally in certain fruits, vegetables, algae, and fungi. It belongs to a large group of pigments known as carotenoids. Tomatoes and tomato-based products can act as natural supporters of lycopene in the human diet. Watermelon, pink grapefruit, pink guava, papaya, and apricots are also other significant sources of lycopene.

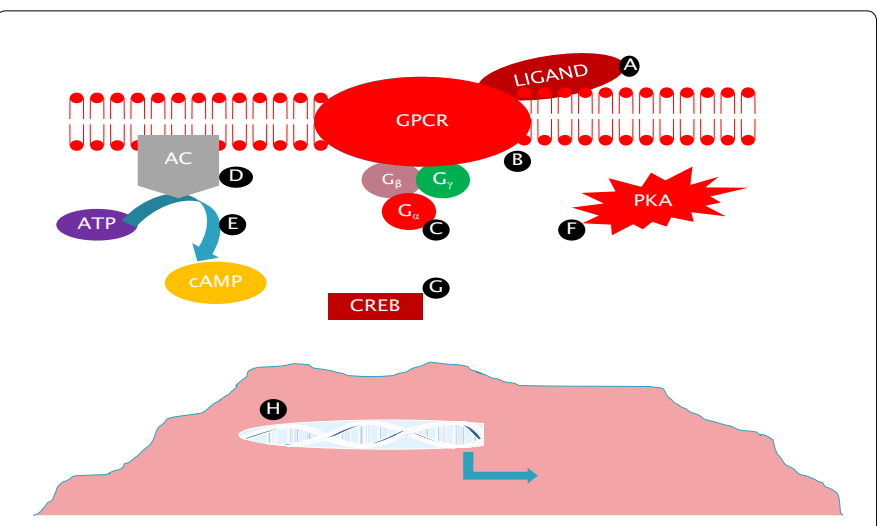

Figure 1: cAMP dependent pathway: (A) Binding of ligand to GPCR; (B) Activation of GPCR; (C) Activation of $G_{\alpha}$ stimulatory protein; (D) Activation of enzyme adenylase cyclase; (E) conversion of ATP to cAMP by enzyme adenylase cyclase; (F) Binding of cAMP to PKA leads to its activation; (G) Activated PKA binds to CREB and phosphorylate it; (H) Activated CREB moves in to the nucleus and enhances the gene expression.

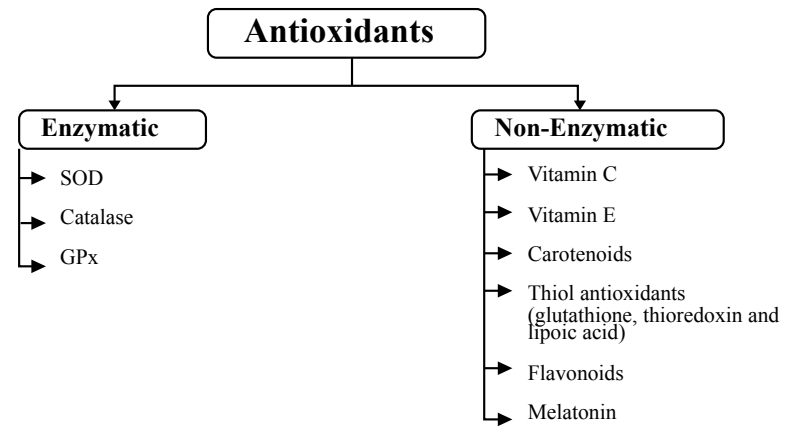

Figure 2: Classification of Antioxidants<smiles>CC(C)=CCC/C(C)=C/C=C/C(C)=C/C=C/C(C)=C/C=C/C=C(C)/C=C/C=C(C)/C=C/C=C(\C)CCC=C(C)C</smiles>

Figure 3: Molecular structure of Lycopene.

Increased ingestion of tomatoes and tomato products containing lycopene has been shown to be associated with decreased risk of chronic diseases including cancer. For example, serum and tissue lycopene levels have been inversely related with prostate cancer risk. Synthetically synthesized lycopene is found as a red to dark violet crystalline powder, it is insoluble in water and nearly insoluble in methanol and ethanol, but is freely soluble in chloroform and tetrahydrofuran, moreover it is sparingly soluble in ether, hexane, and vegetable oils. A solution in hexane shows an absorption maximum at approximately $470 \mathrm{~nm}$ [17]. Although there is significant evidence supporting the actions of lycopene as a potent antioxidant, there are a number of other potential mechanisms through which tomato products providing lycopene and other phytochemicals may reduce the risk for chronic diseases, including common forms of cancer and heart disease. This review will focus on the evidences that lycopene and related carotenoids have 
unexpected biologic effects in experimental systems, some of which may contribute to their observed preventive properties [17]

\section{Diabetes}

Diabetes a metabolic complication is widely affected by the effect of lycopene, it has inverse affect on type-2 diabetes and impaired glucose metabolism. The fact was proven by Coyne et al. [18], that plasma glucose and fasting insulin concentrations lowered down appreciably with raise in serum lycopene. Besides, Polidori et al. [19] showed that plasma lycopene were significantly lesser in very old diabetic patients as compared to controls, while significant converse correlations were found between age and lycopene. In man, dietary lycopene was directly related to baseline serum concentrations of non esterified fatty acids [20]. Moreover, there is also a concern about dietary lycopene and modulation of insulin-like growth factor (IGF). Riso et al. [21] have examined the effect of tomato drink involvement providing small amounts of lycopene and other carotenoids on serum levels of IGF-1. The results indicated that lycopene supplementation before and after each experimental period were inversely and significantly correlated with those of IGF-1. However, Wang et al. have found low evidence for an association between baseline plasma lycopene and the risk of type-2 diabetes in middle-aged and older women after adjustment for multiple risk factors [22].

\section{Cancer}

In the past decade cancer has emerged as a major public health problem around the world. This health issue has raised the awareness of people to go for natural products and their therapeutic or preventive value. The beneficial effect of lycopene is associated to decrease cancer incidence worldwide especially in prostate. Lycopene (1-4 $\mu \mathrm{M})$ was also reported to reduce the risk of prostate, lung, leukemic and digestive tract cancers [23].

Study has reported higher plasma lycopene were inversely associated with prostate cancer risk [24]. Lycopene was able to delay high-grade prostate intraepithelial neoplasia (HGPIN) from developing into prostate cancer and also inversely related to the prostate specific antigen. On the other hand, lycopene $(1-10 \mu \mathrm{M})$ was able to inhibit human liver cancerous cells proliferation and preventing them from metastatic process. Lycopene has significantly inversed the proliferation of human colon carcinoma, chronic lymphocytic leukemia, erythroleukemia and Burkitt lymphoma cell lines [22].

The other antioxidant which will be used in this study is Betalain; it also has many beneficial effects on various medical abnormalities such as LDL oxidation and thalassemia.

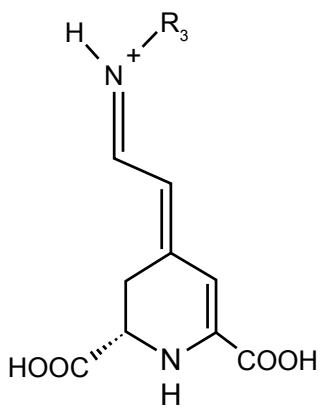

Figure 4: Molecular structure of Betalain
Betalain an unusual and powerful class of antioxidant can be found in roots, fruits and flowers. They absorb visible radiation over the range of $476-600 \mathrm{~nm}$ with a maximum at $537 \mathrm{~nm}$ at $\mathrm{pH}$ 5.0. The suitable for eating known sources of betalain are red and yellow beetroot (Beta vulgaris L. ssp. vulgaris), coloured Swiss chard, grain or leafy amaranth (Amaranthus sp.) and cactus fruits, such as those of Opuntia and Hylocereus genera [25].

The foremost commercially utilised betalain crop is red beetroot (Beta vulgaris), which is comprised of two major soluble pigments, betanin (red) and vulgaxanthine I (yellow). The content of pigments varies depending on the cultivar, although some new varieties produce higher betalain contents. In addition, betalain were able to act as reductants of the redox intermediates of myeloperoxidase, which catalyzes the production of $\mathrm{HClO}$. Lee et al. reported the ability of betalain to induce quinine reductase, a potent detoxification enzyme associated with cancer chemoprevention. Zou et al. reported growth inhibition of cervical, ovarian and bladder cancer cells in vitro and mice ovarian cancer model in vivo by cactus pear extract, whose antioxidant activity was suggested to be partially due to betalain. In vivo tests carried out by Tesoriere et al. suggested that cactus pear fruit decreases oxidative damage to lipids, and improves antioxidant status in healthy humans. Comparative tests with vitamin $\mathrm{C}$ supplementation suggested that some component other than ascorbic acid has helped to decrease lipid oxidation [25].

\section{LDL oxidation}

Oxidation of low-density lipoprotein (LDL) is suggested as a key factor in human atherosclerosis. Oxidatively modified LDL is a potent ligand for scavenger receptors on macrophages and thus contributes to generation of macrophage-derived foam cells, the hall-mark of early atherosclerotic fatty streak lesions. Oxidative modification hypothesis of atherosclerosis is supported by findings, such as the presence of epitopes of oxidatively modified LDL in atherosclerotic lesions and elevated titers of circulating auto-antibodies against oxidized LDL in patients with carotid atherosclerosis. Antioxidant compounds provide resistance to this process and have been suggested to lower atherogenicity. Considerable epidemiologic, biochemical, and clinical evidences support this hypothesis. LDL contains different lipophilic antioxidants, the most abundant being a-tocopherol, the major form of Vitamin E. vitamin E is considered to be the major non-enzymatic antioxidant present in the lipid structures of cells and lipoproteins [26]

\section{Thalassemia}

Beta-thalassemia is a genetic hemolytic disorder characterized by an increased generation of reactive oxygen species, first caused by haemoglobin auto-oxidation and precipitation. This is associated to depletion of the RBC's antioxidant defense, these further results in damage to cells and its components like liver, heart, mitochondria, impairment of morphology and function of cell membrane which results in accelerated RBC destruction. Antioxidant vitamins and phytochemicals may be helpful to treat $\beta$-thalassemia. Protective effects of indicaxanthin on both membrane and soluble compartments of $\beta$-thalassemic RBCs submitted to an in vitro oxidation by cumene hydroperoxide have been demonstrated. The betalain enhanced the resistance to haemolysis, prevented lipid and haemoglobin oxidation, and retarded vitamin E and GSH depletion, dose-dependently. The same work it has been reported that spiking of blood from thalassemia patients with indicaxanthin resulted in its incorporation in the RBCs, indicating that the pathological alterations to the membrane do not affect a trans-bilayer movement of this phytochemical. The finding 
Citation: Tandon S, Kukreja A, Mishra A, Tiwari A (2013) Antioxidants: Potential Inducers of Gene Expression through CAMP Dependent Pathway. J Blood Disorders Transf S3: 006. doi:10.4172/2155-9864.S3-006

that indicaxanthin can be incorporated in the redox machinery of $\beta$-thalassemia RBCs, suggests opportunities of therapeutic interest for this phytochemical [27].

Taking into account the above mentioned studies on the role of antioxidants in the treatment of various diseases, betalain and lycopene both can be used for the treatment of thalassemia to some extent by augmenting the cAMP level which leads to the increased expression of $\gamma$-globin gene in the erythroid cells.

\section{Conclusion}

This review provides an insight on the beneficial effects of two good antioxidants viz. Lycopene and Betalain in preventing various dreaded diseases like cancer, diabetes, oxidative stress etc. above all prime focus is on the role these antioxidants on correcting the molecular defects caused due to $\beta$-thalassemia. Invariably these antioxidants act via multiple biological pathways for the treatment or preventing the diseases. In thalassemia, the main cause is the imbalance of $\beta$-globin chain and hence by using antioxidants the imbalance can be compensated with the over expression of $\gamma$-globin gene. Therefore future research should be focused on balancing the globin genes to cure thalassemia and this can be achieved by augmenting the cAMP level which may lead to the increased expression of $\gamma$-globin gene in the erythroid cells.

\section{Conflict of Interest}

There is no conflict of interest among the authors

\section{References}

1. http://www.thalassemia.com/ (Accessed on 12/12/2012).

2. Pacheco P, Peres MJ, Faustino P, Pischedda C, Gonçalves J, et al. (1995) Beta-thalassaemia unlinked to the beta-globin gene interacts with sickle-cell trait in a Portuguese family. $\mathrm{Br} \mathrm{J}$ Haematol 91: 85-89.

3. Motum PI, Kearney A, Hamilton TJ, Trent RJ (1993) Filipino beta zero thalassaemia: a high $\mathrm{Hb}$ A2 beta zero thalassaemia resulting from a large deletion of the 5' beta globin gene region. J Med Genet 30: 240-244

4. Voet D, Voet JG (2011) Biochemistry: John Wiley \& Sons.

5. Shawky RM, Kamal TM (2012) Thalassemia intermedia: An overview. Egyptian Journal of Medical Human Genetics.13: 245-255.

6. Bailey L, Kuroyanagi Y, Franco-Penteado CF, Conran N, Costa FF, et al. (2007) Expression of the gamma-globin gene is sustained by the cAMP-dependent pathway in beta-thalassaemia. $\mathrm{Br} \mathrm{J}$ Haematol 138: 382-395.

7. Nikolaev VO, Lohse MJ (2006) Monitoring of cAMP synthesis and degradation in living cells. Physiology (Bethesda) 21: 86-92.

8. Nelson DL, Cox MM. Lehninger Principles of Biochemistry Lecture Notebook: W. H. Freeman; 2004.

9. Spina A, Di Maiolo F, Esposito A, D'Auria R, Di Gesto D, et al. (2013) Integrating leptin and cAMP signalling pathways in triple-negative breast cancer cells. Front Biosci (Landmark Ed) 18: 133-144.
10. Bargagli $R$ (2000) Trace metals in Antarctica related to climate change and increasing human impact. Rev Environ Contam Toxicol 166: 129-173.

11. Cadenas E (1989) Biochemistry of oxygen toxicity. Annu Rev Biochem 58: 79110

12. Baynes JW, Thorpe SR (1999) Role of oxidative stress in diabetic complications: a new perspective on an old paradigm. Diabetes 48: 1-9.

13. Devasagayam TP, Tilak JC, Boloor KK, Sane KS, Ghaskadbi SS, et al. (2004) Free radicals and antioxidants in human health: current status and future prospects. J Assoc Physicians India 52: 794-804.

14. Flora SJ (2009) Structural, chemical and biological aspects of antioxidants for strategies against metal and metalloid exposure. Oxid Med Cell Longev 2: 191206.

15. Matés JM, Pérez-Gómez C, Núñez de Castro I (1999) Antioxidant enzymes and human diseases. Clin Biochem 32: 595-603.

16. Uttara B, Singh AV, Zamboni P, Mahajan RT (2009) Oxidative stress and neurodegenerative diseases: a review of upstream and downstream antioxidant therapeutic options. Curr Neuropharmacol 7: 65-74.

17. Olempska Z (2006) Lycopene synthetic synthetic chemical and technical assesment(CTA). US Food and Drug Administration. 1-6.

18. Coyne T, Ibiebele TI, Baade PD, Dobson A, McClintock C, et al. (2005) Diabetes mellitus and serum carotenoids: findings of a population-based study in Queensland, Australia. Am J Clin Nutr 82: 685-693.

19. Polidori MC, Mecocci P, Stahl W, Parente B, Cecchetti R, et al. (2000) Plasma levels of lipophilic antioxidants in very old patients with type 2 diabetes. Diabetes Metab Res Rev 16: 15-19.

20. Ylönen K, Alfthan G, Groop L, Saloranta C, Aro A, et al. (2003) Dietary intakes and plasma concentrations of carotenoids and tocopherols in relation to glucose metabolism in subjects at high risk of type 2 diabetes: the Botnia Dietary Study. Am J Clin Nutr 77: 1434-1441.

21. Riso P, Brusamolino A, Martinetti A, Porrini M (2006) Effect of a tomato drink intervention on insulin-like growth factor (IGF)-1 serum levels in healthy subjects. Nutr Cancer 55: 157-162.

22. Kong KW, Khoo HE, Prasad KN, Ismail A, Tan CP, et al. (2010) Revealing the power of the natural red pigment lycopene. Molecules 15: 959-987.

23. Salman H, Bergman M, Djaldetti M, Bessler H (2007) Lycopene affects proliferation and apoptosis of four malignant cell lines. Biomed Pharmacother 61: 366-369.

24. Zhang J, Dhakal I, Stone A, Ning B, Greene G, et al. (2007) Plasma carotenoids and prostate cancer: a population-based case-control study in Arkansas. Nutr Cancer 59: 46-53.

25. MC Azeredo H (2009) Betalains: properties, sources, applications, and stability - a review. International Journal of Food Science and Technology.44: 23652376.

26. Safari MR, Sheikh N (2003) Effects of flavonoids on the susceptibility of lowdensity lipoprotein to oxidative modification. Prostaglandins Leukot Essent Fatty Acids 69: 73-77.

27. Maria A. Livrea LT (2006) Health Benefits and Bioactive Components of the Fruits from Opuntia ficus-indica. Journal of the Professional Association for Cactus Development. 8: 73-90.
This article was originally published in a special issue, Thrombolic \& Thrombocytopenic Purpurea handled by Editor(s). Dr. Han-Mou Tsai, Pennsylvania State University, USA.
Citation: Tandon S, Kukreja A, Mishra A, Tiwari A (2013) Antioxidants: Potentia Inducers of Gene Expression through CAMP Dependent Pathway. J Blood Disorders Transf S3: 006. doi:10.4172/2155-9864.S3-006 